\title{
Tumor fibroso solitario meníngeo: descripción de tres casos y revisión de la literatura
}

J.M. Saceda-Gutiérrez; A.J. Isla-Guerrero; C. Pérez-López; R. Ortega-Martínez; A. Gómez de la Riva; M.L. GandiaGonzález; M. Gutiérrez-Molina* y J.A. Rey-Herranz**

Servicios Neurocirugía, Anatomía Patológica* y Biología Molecular**. Hospital Universitario La Paz. Madrid.

\section{Resumen}

Presentamos 3 pacientes con tumor fibroso solitario de localización meníngea donde describimos el estudio histológico, así como la evolución después del tratamiento quirúrgico. Los pacientes descritos tenían edades de 37, 52 y 65 años y tras la resección total no se ha objetivado signo de recidiva en ningún caso después de 4, 6 y 7 años de seguimiento respectivamente.

Revisando la literatura se trata de un tumor indistinguible clínica y radiológicamente del meningioma típico, haciendo necesario el uso de pruebas inmunohistoquímicas para realizar el diagnóstico diferencial, donde la positividad para el CD34 y la negatividad para el EMA definen al tumor fibroso solitario. Se trata de un tumor benigno, en el que la resección total es el principal factor pronóstico; no obstante, se han descrito casos de recidivas locales y metástasis a distancia. Todas estas características las encontramos en los casos presentados en el presente trabajo, quedando la incertidumbre de su capacidad de recidiva local o sistémica en el futuro.

PALABRAS CLAVE: Tumores de sistema nervioso central. Tumores meníngeos. Tumor fibroso solitario. Neoplasias células fusiformes. CD34.

Solitary fibrous tumors of the meninges: report of three cases and literature review

\section{Summary}

We report 3 patients with fibrous solitary tumor of meningeal location where we described the histological study, as well as evolution after the surgical treatment. The described patients presented ages of 37, 52 and 65 years, after the resection has not appeared an objective sign of recurrence in any case after 4, 6 and 7 years of follow-up respectively.

Recibido: 2-01-07. Aceptado: 10-04-07
Checking the literature the tumor is indistinguishable clinical and radiolocally of the typical meningioma, doing necessary the use of inmunohistochemistry to do the differential diagnosis, where positiveness for CD34 and the negativeness for EMA define the fibrous solitary tumor. It is about a benign tumor, where total removing is the principal factor in prognosis, nevertheless there are cases of local recurrences and long-distance metastasis. We can find all these characteristics in the showed cases of the present article, having the uncertainty of its local or systemic relapse ability in the future.

KEY WORDS: Central nervous system tumors. Meningeal tumors. Solitary fibrous tumor. Spindle cell neoplasm. CD34.

Introducción

El tumor fibroso solitario (TFS) es una neoplasia de origen mesenquimal constituida por células fusiformes ${ }^{29}$. Fue descrito por primera vez en 1931 por Klemperer y Rabin con una localización típicamente pleural visceral ${ }^{18}$. Han sido descritos casos de localización extrapleural: pericardio, peritoneo, mediastino, pulmón, hígado, cavidad nasal, tiroides y meninges ${ }^{12,13,22,39,45}$.

Han pasado 10 años desde que en 1996, con la descripción por parte de Carneiro y cols. ${ }^{5}$ de siete casos de TFS meníngeo, se mostrara al tumor fibroso como una entidad propia que podía ser diferenciado por características histológicas e inmunohistoquímicas del clásico meningioma fibroso.

La mayoría de TFS tienen unas características histológicas benignas, pero se han descrito casos de recidivas locales e incluso un caso de metástasis pulmonar tras un largo periodo de tiempo ${ }^{16,33}$. El diagnóstico se basa en el perfil inmunohistoquímico, siendo muy positivo para

Abreviaturas. EMA: antígeno epitelial de membrana. HPC: hemangiopericitoma. TC: tomografía computarizada. TFS: tumor fibroso solitario. RM: resonancia magnética. 

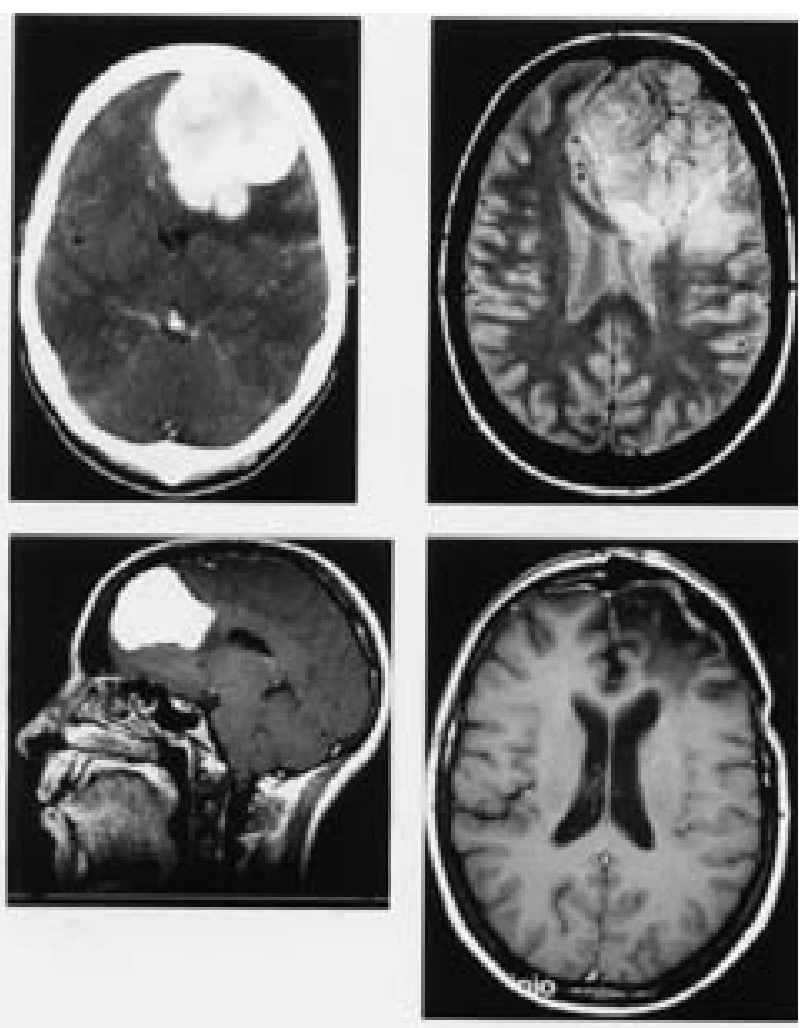

Figura 1. Imágenes de TC craneal y de RM craneal, mostrando una gran tumoración frontal izquierda captadora de contraste con edema vasogénico perilesional.

Figura 1 (abajo derecha). RM control 4 años después de la intervención quirúrgica, sin evidencia de recidiva tumoral.

CD34, vimentina, bcl-2 y CD99, pero negativo para los marcadores EMA y S- $100^{6}$.

\section{Material y métodos}

Hemos hallado tres casos de TFS meníngeo que fueron intervenidos quirúrgicamente en nuestro centro con una resección total de la lesión. Todos ellos tuvieron un diagnóstico, previo a la confirmación histológica, de posible meningioma. Macroscópicamente todos ellos eran de consistencia sólida, con una dura cápsula fibrosa y en algunos se podía apreciar algún foco hemorrágico; microscópicamente se les realizó la batería de pruebas inmunohistoquímicas con resultados positivos para CD34 $\mathrm{y}$ vimentina, $\mathrm{y}$ negativa para EMA.

\section{Casos}

\section{Caso I}

Mujer de 37 años que refería cefalea a nivel frontal
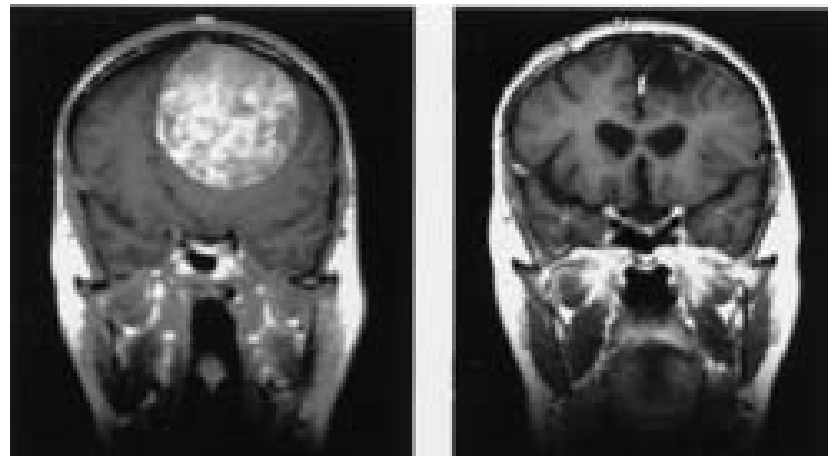

Figura 2 (izquierda). Imagen de RM que mostraba una masa extraaxial de implantación en la hoz captadora de contraste.

Figura 2 (derecha). RM control 6 años después de la intervención quirúrgica

izquierdo de tres semanas de evolución, de inicio brusco y no irradiada; además acompañaba sensación de acorchamiento, de tres días de evolución, en hemilengua izquierda que se fue extendiendo a toda la hemicara ipsilateral. En la exploración física, se encontró edema de papila bilateral e hipoestesia superficial en hemicara izquierda. El resto de la exploración neurológica fue normal.

En la tomografía computarizada (TC) craneal se evidenció una lesión extraaxial frontal izquierda con moderado efecto masa e importante edema acompañante; los hallazgos en resonancia magnética (RM) mostraron un proceso expansivo en casi todo el lóbulo frontal que captaba contraste de forma importante; en secuencia T2 se confirmó la existencia de vasos intratumorales y un importante edema que producía herniación subfalciana. (Figura 1).

En la intervención quirúrgica se realizó una craneotomía frontal izquierda con resección completa de la pétrea tumoración blanquecino-amarillenta y de la superficie dural a la que estaba adherida. La paciente evolucionó favorablemente, encontrándose actualmente asintomática y sin signos radiológicos de recidiva 4 años después. (Figura 1, abajo derecha).

\section{Caso 2}

Mujer de 52 años, sin antecedentes de interés que desde hacía dos meses presentaba cefalea holocraneal que cedía parcialmente con analgésicos. En la exploración neurológica se apreció únicamente edema de papila bilateral.

En la TC se encontró una tumoración de gran tamaño, que captaba contraste de forma homogénea, implantada en el tercio medio del seno longitudinal; la RM mostró una masa extraaxial de implantación en la hoz y seno longitudinal. (Figura 2, izquierda); en la arteriografía se observó un bloqueo del seno longitudinal a nivel del tercio 


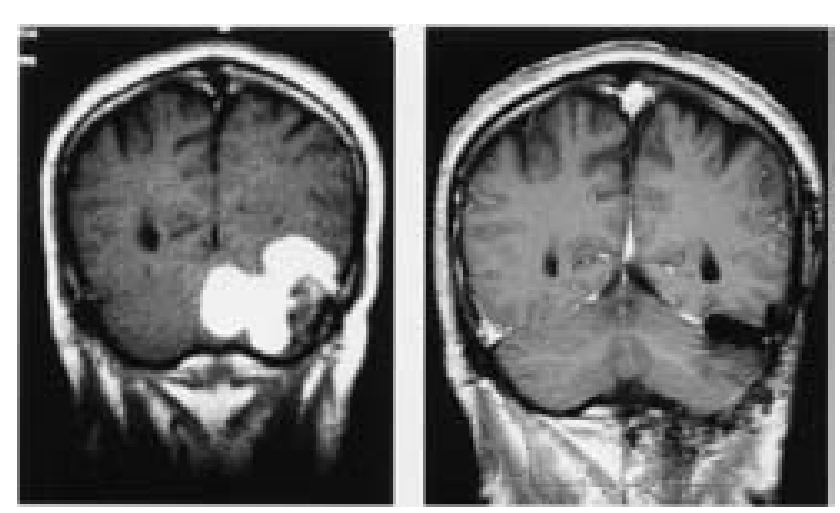

Figura 3 (izquierda). Imagen de RM que mostraba un tumor tentorial izquierdo que capta contraste.

Figura 3 (derecha). RM control 7 años después de la intervención quirúrgica.

medio.

En la intervención quirúrgica se realizó una craneotomía frontal izquierda con resección total de la lesión y de la porción del seno longitudinal afectada. La paciente se encuentra asintomática 6 años después de la intervención. (Figura 2, derecha).

\section{Caso 3}

Varón de 65 años que desde hacía varios meses presentaba cefalea con sensación de calor en región occipital. Este dolor se incrementaba con el movimiento cefálico y era de carácter continuo. En la exploración no existía ningún signo de focalidad neurológica.

La TC mostró una lesión intracraneal expansiva compatible con meningioma supra e infratentorial izquierdo con signos de herniación transtentorial ascendente; la RM objetivó un proceso expansivo supra e infratentorial adherido a tentorio izquierdo (Figura 3, izquierda) y en la angiografía cerebral se observó trombosis del seno lateral izquierdo.

En la intervención quirúrgica se practicó una craneotomía occipital izquierda y de fosa posterior, extirpándose un voluminoso proceso expansivo, junto con parte de tentorio al que estaba adherido y del seno lateral, el cual estaba parcialmente trombosado. El paciente se encuentra asintomático 7 años después de la cirugía. (Figura 3, derecha)

\section{Histopatología}

En las imágenes histológicas de los tres casos presentados podíamos encontrar: proliferación tumoral constituida por células fusiformes con núcleo ovalado y citoplasma eosinófilo que tendían a disponerse en fascículos (Figura 4 a). No se observaron atipias ni mitosis. Se reconocieron
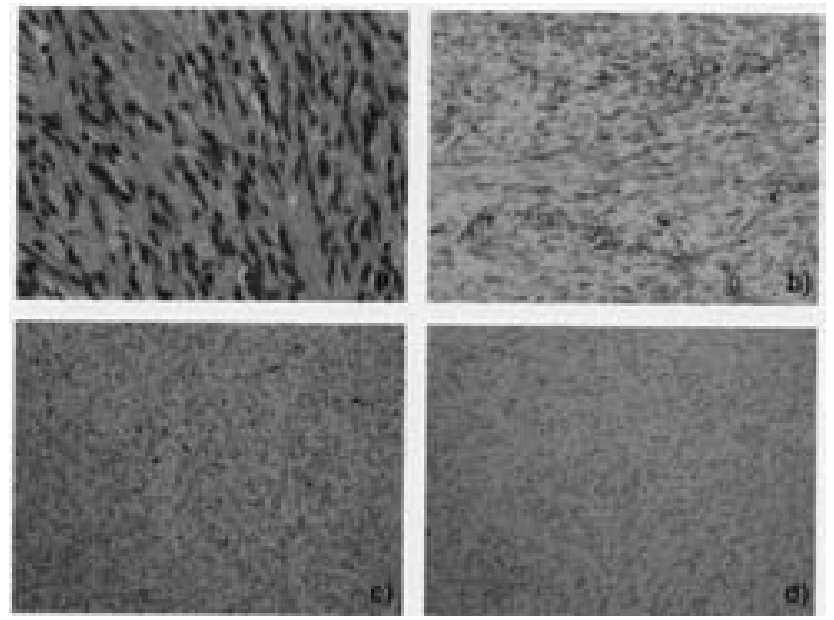

Figura 4 a. Tinción con Hematoxilina-Eosina, apreciando las células fusiformes características.

Figura 4 b. Gran inmunorreactividad para CD34.

Figura 4 c. Bajo índice proliferativo $(<5 \%)$.

Figura 4 d. Negatividad para el antígeno epitelial de membrana.

fragmentos de tejido conjuntivo bien organizado que se interpreta de origen paquimeníngeo. Las células tumorales expresaban positividad para CD34 (Figura 4 b) con un índice de proliferación, expresado con Ki-67, menor de un 5\% (Figura 4 c). Las células presentaban negatividad para EMA (Figura 4 d), S-100 y proteína gliofibrilar.

\section{Discusión}

Desde la primera descripción por parte de Carneiro hasta la fecha, se han descrito, aproximadamente 50 casos de TFS con diversas localizaciones intracraneales ${ }^{12,13,22,39,45}$. También se han encontrado 15 casos de TFS de localización espinal.

El diagnóstico se basa en pruebas inmunohistoquímicas, ya que la clínica y la imagen radiológica, con $\mathrm{TC}$ y $\mathrm{RM}$, mimifica la de un meningioma ${ }^{31}$; entre las pruebas histológicas, destacan la positividad muy marcada para CD34 (antígeno descrito originariamente en células madre hematopoyéticas) y la negatividad para EMA. Todos estos hallazgos orientan a su origen mesenquimal ${ }^{36}$.

\section{Epidemiología}

En los casos de TFS descritos en la literatura, atendiendo a la distribución por edad y sexo, existe un ligero predominio masculino en torno al final de la quinta década. Si analizamos la zona anatómica donde surge la lesión, se han descrito casos en prácticamente toda la superficie endocraneal, pero parece existir una predisposición por la fosa posterior y la zona frontal (tabla 1). La mayor parte 
Tabla 1

Casos recogidos en la literatura de TFS

\begin{tabular}{|c|c|c|c|c|c|c|}
\hline Caso & Edad/sexo & Historia & Localización & Resección & Recidiva & Seguimiento \\
\hline \multirow[t]{5}{*}[7]{} & $51 / \mathrm{F}$ & $\begin{array}{l}\text { Hemiparesia } \\
\text { Cefalea, disfagia }\end{array}$ & Fosa post. & Total & No & 20 años \\
\hline & $47 / \mathrm{M}$ & Visión borrosa & APC der. & Total+Rdt & No & 10 años \\
\hline & $73 / \mathrm{M}$ & Cefalea & Frontal izq. & Total & No & 8 meses \\
\hline & $62 / \mathrm{F}$ & Cefalea, vértigo & $\begin{array}{l}\text { Tentorio izq. y } \\
\text { supratentorial }\end{array}$ & Total & No & 7 meses \\
\hline & $63 / \mathrm{F}$ & $\begin{array}{l}\text { Hipoacusia y } \\
\text { parestesia facial }\end{array}$ & APC der.. & Total & No & 9 meses \\
\hline [19] & $73 / \mathrm{F}$ & No conocida & $\begin{array}{l}\text { Canal óptico } \\
\text { der. }\end{array}$ & Total & $\begin{array}{c}\text { No } \\
\text { conocida }\end{array}$ & No conocido \\
\hline [37] & $43 / \mathrm{M}$ & $\begin{array}{l}\text { Pérdida de } \\
\text { memoria y visión }\end{array}$ & $\begin{array}{l}\text { Convexidad } \\
\text { frontal izq. }\end{array}$ & Total & $\begin{array}{c}\text { No } \\
\text { conocida }\end{array}$ & No conocido \\
\hline$[36]$ & 3 casos & $\mathrm{NC}$ & $\mathrm{NC}$ & $\mathrm{NC}$ & $\mathrm{NC}$ & $\mathrm{NC}$ \\
\hline [38] & $11 / M$ & $\begin{array}{l}\text { Nódulo dural } \\
\text { asintomático }\end{array}$ & Occipital izq. & Total & $\mathrm{NC}$ & $\mathrm{NC}$ \\
\hline$[46]$ & $45 / \mathrm{M}$ & $\begin{array}{l}\text { Disfunción } \\
\text { autonómica }\end{array}$ & $4^{\circ}$ ventrículo & $\mathrm{NC}$ & No & 15 años \\
\hline$[10]$ & $42 / \mathrm{M}$ & $\begin{array}{l}\text { Hemianopsia } \\
\text { Diplopia }\end{array}$ & $\begin{array}{l}\text { Supraselar } \\
\text { izq }\end{array}$ & $\mathrm{NC}$ & No & 1 año \\
\hline$[4]$ & $30 / \mathrm{M}$ & Cefalea & Frontal der. & $\mathrm{NC}+\mathrm{Rdt}$ & No & 9 meses \\
\hline$[15]$ & $64 / F$ & Epilepsia & Hoz cerebral & Total & $\mathrm{Si}$ & $\begin{array}{l}\text { Muerte } \\
\text { postoperatoria }\end{array}$ \\
\hline [33] & $14 / \mathrm{F}$ & $\begin{array}{l}\text { Cefalea y } \\
\text { pérdida de visión }\end{array}$ & $\begin{array}{l}\text { Convexidad } \\
\text { parietal der. }\end{array}$ & Total & $\mathrm{NC}$ & $\mathrm{NC}$ \\
\hline [31] & $58 / \mathrm{F}$ & $\begin{array}{l}\text { Ataxia e } \\
\text { hipoacusia }\end{array}$ & $\begin{array}{l}\text { Fosa post. } \\
\text { lateral der. }\end{array}$ & Total & No & $\mathrm{NC}$ \\
\hline [32] & $55 / \mathrm{F}$ & $\mathrm{NC}$ & $\begin{array}{l}\text { Fosa post. } \\
\text { der. }\end{array}$ & Total $+\mathrm{Rdt}$ & $\begin{array}{c}\mathrm{Si} \\
\text { (pulmón) }\end{array}$ & 11 meses \\
\hline [43] & $60 / \mathrm{F}$ & $\mathrm{NC}$ & Frontal & $\mathrm{NC}$ & $\mathrm{NC}$ & 18 meses \\
\hline [27] & $51 / \mathrm{F}$ & $\mathrm{NC}$ & Seno transv. & Subtotal & $\mathrm{Si}(15 \mathrm{a})$ & 7 años \\
\hline & $54 / \mathrm{F}$ & $\mathrm{NC}$ & Pariet-occip & Total & No & 3 años \\
\hline
\end{tabular}




\begin{tabular}{|c|c|c|c|c|c|c|}
\hline Caso & Edad/sexo & Historia & Localización & Resección & Recidiva & Seguimiento \\
\hline [43] & $61 / F$ & $\mathrm{NC}$ & Frontal & Subtotal+rdt & No & 10 meses \\
\hline \multirow[t]{4}{*}[25]{} & $46 / F$ & Cefalea & Fosa lat. post & Total & No & 3 años \\
\hline & $43 / \mathrm{M}$ & Cefalea & APC & Subtotal & No & 2 años \\
\hline & $72 / \mathrm{F}$ & $\begin{array}{l}\text { Hemiparesia } \\
\text { y disfasia }\end{array}$ & $\begin{array}{l}\text { Fosa media } \\
\text { izq. }\end{array}$ & Subtotal & No & 1 año \\
\hline & $71 / \mathrm{M}$ & $\begin{array}{l}\text { Hemorragia } \\
\text { intracerebral }\end{array}$ & $\begin{array}{l}\text { Frontoparietal } \\
\text { bilateral }\end{array}$ & Incompleta & - & $\begin{array}{l}\text { Muerte } \\
\text { postoperatoria }\end{array}$ \\
\hline$[1]$ & $58 / \mathrm{M}$ & $\mathrm{NC}$ & Temporal & Total & No & 1 año \\
\hline [9] & $25 / \mathrm{F}$ & $\mathrm{NC}$ & Occipital & Total & No & 3 años \\
\hline [41] & 12 casos & $\mathrm{NC}$ & $\begin{array}{l}9 \text { supratentorial/ } \\
3 \text { infratentorial }\end{array}$ & - & - & - \\
\hline \multirow[t]{3}{*}[8]{} & $38 / \mathrm{M}$ & $\mathrm{NC}$ & Ganglio gasser & Total & No & 15 meses \\
\hline & $29 / F$ & $\mathrm{NC}$ & Cerebelo & Total & No & 3 años \\
\hline & $34 / \mathrm{M}$ & $\mathrm{NC}$ & Frontal & Total & No & 5,5 años \\
\hline [21] & $63 / \mathrm{M}$ & $\begin{array}{l}\text { Cefalea y } \\
\text { debilidad MMII }\end{array}$ & Intraventricular & Subtotal & No & 3,5 años \\
\hline [23] & $50 / \mathrm{M}$ & $\mathrm{NC}$ & Paraselar & $\mathrm{NC}$ & $\mathrm{NC}$ & $\mathrm{NC}$ \\
\hline$[35]$ & $50 / \mathrm{M}$ & Cefalea & Tentorial & Total & No & 6 meses \\
\hline [28] & $48 / \mathrm{F}$ & $\mathrm{NC}$ & $\begin{array}{l}\text { Falcio - } \\
\text { tentorial }\end{array}$ & Total & $\begin{array}{l}\mathrm{Si}(15 \mathrm{a} \\
\text { después) }\end{array}$ & $\mathrm{NC}$ \\
\hline [20] & $44 / F$ & $\begin{array}{l}\text { Cefalea y } \\
\text { alt. marcha }\end{array}$ & Tentorial & Total & $\begin{array}{l}\text { Si }(5 \text { veces } \\
\text { local y mts } \\
\text { pulmonar } \\
\text { en } 26 a)\end{array}$ & \\
\hline [24] & $33 / \mathrm{M}$ & $\begin{array}{l}\text { Disminución } \\
\text { visión }\end{array}$ & Paraselar & $\begin{array}{c}\text { Subtotal } \\
+\mathrm{Rdt}+\text { Qmt }\end{array}$ & No & $\mathrm{NC}$ \\
\hline \multirow[t]{2}{*}{ [17] } & $49 / F$ & Cefalea & $4^{\circ}$ ventriculo & Subtotal & $\mathrm{NC}$ & $\mathrm{NC}$ \\
\hline & $30 / \mathrm{M}$ & Cefalea & Foramen yug & $\mathrm{NC}$ & $\mathrm{NC}$ & $\mathrm{NC}$ \\
\hline [34] & $\mathrm{NC}$ & $\mathrm{NC}$ & Seno lateral & Total & No & $\mathrm{NC}$ \\
\hline$[3]$ & $55 / \mathrm{F}$ & $\mathrm{NC}$ & Seno transverso & Total & $\mathrm{Si}(2,5 \mathrm{a})$ & $\mathrm{NC}$ \\
\hline \multirow[t]{2}{*}{ [26] } & $12 / \mathrm{M}$ & Hemiparesia & Parietal & Total & No & 3,5 años \\
\hline & $\begin{array}{l}\text { Nuestro } \\
\text { estudio }\end{array}$ & 3 casos & & & & \\
\hline
\end{tabular}

APC: ángulo pontocerebeloso; RDT: radioterapia; QMT: quimioterapia. MTS: metástasis; Foramen yug: foramen yugular; NC: No conocido (falta de datos en artículo revisado) 
Tabla 2

Diagnóstico diferencial tumoral en estudio histológico

\begin{tabular}{|l|c|c|c|c|c|}
\hline Tumor/inmunohist. & CD34 & Vimentina & BCL-2 & EMA & Factor Xllla \\
\hline T. fibroso & +++ & +++ & + - & - & - \\
\hline Meningioma & - & + & - & + & - \\
\hline Hemagiopericitoma & $+(33-100 \%)$ & $+(80 \%)$ & - & - & + \\
\hline
\end{tabular}

de los TFS intracraneales tienen un claro origen dural, no ocurriendo así con la mayoría de los TFS espinales.

\section{Estudios radiológicos}

En la RM cerebral encontraremos una estructura bien definida con señal isodensa en T1 que capta homogéneamente gadolinio y señal hipodensa en T2, respecto al líquido cefalorraquídeo; en la secuencia Flair podemos encontrar señales hipo e hiperintensas intralesionales que suelen corresponder con zonas ricas en colágeno o, como ocurre en nuestro primer caso, con vascularización intratumoral, así como realce del edema perilesional (figura 1). También nos podemos encontrar zonas de engrosamiento dural y erosión de la tabla interna. Por lo tanto, no existe ningún signo radiológico específico de TFS que lo distinga del meningioma típico ${ }^{21,23}$.

\section{Genética}

En el análisis cromosómico del tumor fibroso solitario meníngeo se han escrito alteraciones como la pérdida del cromosoma 3 y deleciones en la región 3p21 p26. Otras pérdidas descritas son deleción 4p15, 8q22q24, 10, $11 q 14 q 25,20$ y 21. También se han encontrado ganancias en $18 \mathrm{p} 11 \mathrm{p} 13,1 \mathrm{p} 11 \mathrm{p} 361$ p13 y 20q11q13.

Una pequeña proporción de meningiomas presentaron cambios en el cromosoma 3 , sobre todo 3 p12, no existiendo evidencia de semejanza genética entre meningioma y TFS.

Si analizamos el estudio cromosómico de tumor fibroso solitario no meníngeo, se han encontrado anomalías en el $50 \%$ de los casos, sobre todo pérdida de cromosoma 13 y trisomía 8, no pudiendo encontrar correlación entre el cariotipo, histología tumoral, localización o pronóstico ${ }^{2}$.

\section{Histopatología diferencial con meningioma y heman- giopericitoma}

Debido a la similitud macroscópica del TFS con otros procesos de mayor prevalencia, casi todos los casos son diagnosticados a posteriori por estudio histológico.

La pieza clave para el diagnóstico son las pruebas inmunohistoquímicas. El TFS es marcadamente positivo para vimentina y CD34 (glucoproteína transmembrana que típicamente se encuentra en células madre hematopoyéticas); hay que tener en cuenta que el CD34 no es exclusivo de TFS, ya que de forma parcheada puede ser hallada en hemangiopericitoma (HPC), meningioma fibroso y neurofibroma (Tabla 2). Otro componente importante para el diagnóstico de TFS es la reactividad positiva contra la proteína antiapoptótica bcl-2 $2^{11,36}$. Recientemente se ha descrito la positividad para CD $117^{3}$.

Es importante conocer la negatividad del TFS para marcadores de cresta neural como son S-100, GFAP, EMA, antígenos vasculares o citoqueratina.

Todo ello es importante para distinguir los procesos que puedan requerir tratamiento postquirúrgico adyuvante, como radioterapia.

\section{Malignización}

El mayor número de casos estudiados de tumor fibroso son de origen extracraneal, concretamente de origen pleural; en la gran mayoría de éstos, los hallazgos histológicos orientan a un comportamiento benigno; no obstante, se han descrito signos de atipia: como hipercelularidad, pleomorfismo nuclear, aumento de mitosis y necrosis ${ }^{14}$. Estas características histológicas pueden correlacionarse con recurrencia local o diseminación a distancia ${ }^{42}$; ésta última afirmación no es extrapolable al TFS meníngeo.

En la literatura acerca de la agresividad biológica del TFS existen cuatro casos de recurrencia local ${ }^{15,27,17,34}$, uno de local, otro líquido cefalorraquídeo ${ }^{28}$ y dos de extensión pulmonar $^{20,33}$.

Si queremos intentar predecir el comportamiento que va a tener el tumor, podemos establecer una serie de principios, basados en la experiencia de la literatura, como son: el índice mitótico y la expresión de Ki-67 parecen aumentar paulatinamente conforme el tumor tiende a recidivar, siendo el tiempo entre las recurrencias cada vez menor. No 
parece existir ningún signo histológico específico que nos oriente acerca de la capacidad del tumor de recidivar localmente o a distancia, ya que, incluso en presencia de signos de malignidad, la evolución es poco agresiva ${ }^{20}$.

Las imágenes histológicas del HPC pueden ser confundidas con las del TFS con rasgos de malignidad; de hecho, se admite que pequeños focos de HPC pueden aparecer en TFS, sugiriendo la posibilidad de ser ambos procesos variantes de la misma entidad ${ }^{30,44}$.

\section{Pronóstico}

Tenemos en la literatura casos de TFS libre de recurrencia de más de 25 años $^{7}$, casos de recurrencia local a los 2 años y medio ${ }^{3}$ e incluso dos casos de diseminación a distancia 20 años después del primer diagnóstico.

Atendiendo al mayor tiempo de experiencia con el TFS pleural, se puede concluir que la resección total es mejor índice predictor que el grado de malignidad histológica; como ocurre en nuestros tres casos, donde tras una completa eliminación de la lesión los pacientes llevan cuatro, seis y siete años libres de enfermedad. Por lo tanto, parece existir unanimidad en el manejo inicial y de las recidivas mediante extirpación quirúrgica total. El papel de la radioterapia como tratamiento adyuvante es desconocido debido a la escasez de casos con atipias histológicas o de recidivas locales $^{20}$.

Nos encontramos ante una entidad ya bien establecida, pero sin un conocimiento certero de su comportamiento con los años debido a que las recidivas descritas ocurren con demora de hasta varias décadas.

\section{Conclusión}

En este artículo aportamos tres nuevos casos de TFS a la literatura. Los hallazgos encontrados están en consonancia con los datos previamente conocidos desde que hace diez años se hiciese la primera reseña bibliográfica de esta entidad patológica.

Parece haber unanimidad en el hecho de que el tumor fibroso tiene un componente histológico benigno y si va acompañado de una resección quirúrgica completa el pronóstico es bueno; sin embargo, han sido descritos casos de recidiva local y menos frecuentemente a distancia, hecho que de producirse, ocurre casi siempre con una demora de hasta varias décadas.

Todo esto sirve para confirmar la existencia de un nuevo tipo histológico de tumor. Aunque ya se hayan descrito cerca de cincuenta casos, aún queda mucho por detallar acerca de la biología tumoral, por lo que es necesario hacer revisiones de los casos publicados en la literatura y ver su comportamiento con los años.

\section{Bibliografía}

1. Ahn, J.Y., Shim, J.Y., Yang, W.I.Y., Kim, T.S.: Meningeal solitary fibrous tumor as an unusual cause of exophthalmos. Case report and review of the literature. Neurosurgery 2001; 48: 1362-1366.

2. Andrew, J.M., Summersgill, B.M., Fisher, C., Shipley, J.M., Dean, A.F.: Chromosomal imbalances in meningeal solitary fibrous tumors. Cancer Genetics and Cytogenetics 2002; 135: $160-164$.

3. Bikmaz, K., Cosar, M., Kurtkaya-Yapicier, O., Iplikcioglu, A.C., Gokduman, C.A.: Recurrent solitary fibrous tumour in the cerebellopontine angle. J Clin Neurosci. 2005 Sep; 12: 829-832.

4. Brunnemann, R.B., Ro, J.Y., Ordonez, N.G., Mooney, J., El-Naggar, A.K., Ayala, A.G.: Extrapleural solitary fibrous tumour: a clinicopathologic study of 24 cases. Mod Pathol 1999; 12: 1034-1042.

5. Carneiro, S.S., Scheithauer, B.W., Nascimento, A.G., Hirose, T.N., Davis, T.H.: Solitary fibrous tumor of the meninges: a lesion distinct from fibrous meningioma. A clinicopathologic and immunohistochemical study. Am J Clin Pathol 1996; 106: 217-224.

6. Carneiro, S.S., Scheithauer, B.W., Nascimento, A.G., Hirose, T., Davis, D.H.: Solitary fibrous tumor of the meninges: a lesion distinct from fibrous meningioma. A clinicopathologic and immunohistochemical study. Am J Clin PathoI1996; 106: 217-224.

7. Carneiro, S.S., Scheithauer, B.W., Nascimento, A.G., Hirose, T., Davis, D.H.: Solitary fibrous tumor of the meninges: a lesion distinct from fibrous meningioma. A clinicopathologic and immunohistochemical study. Am J Clin Pathol 1996; 106: 217-224.

8. Carola, E., Salvati, M., Ramundo Orlando, E., Lenzi, J., Santero, A., Giangaspero, F.: Solitary fibrous tumors of the meninges. Report of four cases and literature review. Neurosurg Rev 2004; 27: 246-251.

9. Centeno, R.S., Gorgulho Pedroso, A.A., Pereira, E.M., Neta, A.R.: Tumor fibroso solitario da meninge. Relato de Caso Arq. Neuro-Psyquiatr 2002; 60: 1-8.

10. Challa, V.R., Kilpatrick, S.E., Ricci, P., Wilson, J.A., Kelly, D.L.: Solitary fibrous tumor of the meninges. Clin Neuropathol 1998; 17: 73-78.

11. Chaubal, A., Paetau, A., Zoltick, P., Miettinen, M.: CD34 inmunoreactivity in nervous system tumors. Acta Neuropathol 1994; 88: 454-458.

12. Dorfman, D.M., To, K., Dickersin, G.R., Rosenberg, A.E., Pilch, B.Z.: Solitary fibrous tumor of the orbit. Am J Surg Pathol 1994; 18: 281-287.

13. El-Naggar, A.K., Ro, J.Y., Ayala, A.G., Ward, R., Ordonez, N.G.: Localized fibrous tumor of the serosal cavities. Am J Clin Pathol 92: 561-565 Nascimiento AG: solitary fibrous tumor. A ubiquitous neoplasm of mesenquimal differentiation. 
Adv Anat Pathology 1996: 388-395.

14. England, D.M., Hochhlolzer, L., Mc Carhy, M.J.: Localized benign and malignant fibrous tumors of the pleura. Am J Surg Pathol 1989; 13: 640-658.

15. Gentil Perret, A., Mosnier, J.F., Duthel, R., Brunon, J., Barral, F., Boucheron, S.: Solitary fibrous tumor of the meninges (in French). Ann Pathol 1999; 19: 532-535.

16. Hasegawa, T., Matsuno, Y., Shimoda, T., Hasegawa, F., Sano, T., Hirohashi, S.: Extrathoracic solitary fibrous tumors. Their histological variability and potentially aggressive behaviour. Hum Pathol 1999; 30: 1464-1473.

17. Kim, K.A., Gonzalez, I., McComb, J.G., Giannotta, S.L.: Unusual presentations of cerebral solitary fibrous tumor. Report of four cases. Neurosurgery 2004; 54 : 1004-1009.

18. Klemperer, P., Rabin, C.B.: Primary neoplasm of the pleura. A report of five cases. Arch Pathol 1931; 11: 385-412.

19. Kuchelmeister, K., Scheuerle, A., Bohle, R.M., Kretschmer, T., Richter, H.P., Schachenmayr, W.: Solitary fibrous tumor - a new differential diagnosis in surgical neuropathology cases. Clin Neuropathol 1997; 16: 270 (Abstract).

20. Kumiko Ogawa, Toyohiro Tada, Satoru Takahashi, et al.: Malignant solitary fibrous tumor of the meninges. Virchows Arch 2004; 444: 459-464.

21. Kocak, A., Cayli, S.R., Sarac, K., Aydin, N.E.: Intraventricular solitary fibrous tumor: an unusual tumor with radiological, ultrastructural, and immunohistochemical evaluation. Case report. Neurosurgery. 2004 Jan; 54: 213-216; discussion 216-217.

22. Kottke-Marchant, K., Hart, W.R., Broughton, T.: Localised fibrous tumor (localised fibrous mesothelioma) of the liver. Cancer 1999; 64: 1096-1102.

23. Lo, C.P., Chen, C.Y., Lin, C.K., Chin, C.K., Juan, C.J., Hsueh, C.J.: Parasellar solitary fibrous tumor of meninges. Resonance magnetic imagin features with pathologic correlation.

24. Macfarlane, R.G., Galloway, M., Plowman, P.N., Thomas, D.G.: A highly vascular intracranial solitary fibrous tumor treated with radiotherapy and toremifene. Case report. Neurosurgery. 2005 Jun; 56 (6).

25. Martin, A.J., Fisher, C., Igbaseimokumo, U., Jarosz, J.M., Dean, A.F.: Solitary fibrous tumors of the meninges. Case series and literature review. J Neurooncol 2001; 54: 57 69.

26. Meagher-Villemure, K., Agazzi, S., Rilliet, B.: Meningeal solitary fibrous tumour in a childChilds Nery Syst. 2006 Jun; 22: 619-622. Epub 2006 Jan 14. de Ribaupierre S.

27. Morimitsu, Y., Masayoshi, N., Masanori, H., Hiroshi, H.: Extrapleural solitary fibrous tumor: clinicopathologic study of 17 cases and molecular analysis of the p53 pathway. Acta Pathol Microbiol Immunol Scand 2000; 108: 617-625.

28. Miyashita, K., Hayashi, Y., Fujisawa, H., Hasegawa, M., Yamashita, J.: Recurrent intracranial solitary fibrous tumor with cerebrospinal fluid dissemination. Case report. J Neuro- surg 2004 Dec; 101:1045-1048.

29. Nascimiento, A.G.: Solitary fibrous tumor. A ubiquitous neoplasm of mesenquimal differentiation. Adv Anat Pathology 1996; 3: 388-395.

30. Nappi, O., Ritter, J.H., Pettinato, G., Wick, M.R.: Hemangiopericytoma. Histological pattern or clinicopathologic entity? Semin Diagn Pathol 1995; 12: 221-232.

31. Nawashiro, H., Nagakawa, S., Osada, H., et al.: Solitary fibrous tumor of the meninges in the posterior cranial fossa. Magnetic resonance imaging and histological correlation. Case report. Neurol Med Chir (Tokyo) 2000; 40: 432434.

32. Nikas, D.C., De Girolami, U., Folkerth, R.D., Bello, L., Zamani, A.A., Black, P.McL.: Parasagittal solitary fibrous tumor of the meninges. Case report and review of the literature. Acta Neurochir (Wien) 1999; 141: 307-313.

33. Ng, H.K., Choi, P.C.L., Wong, C.W., To, K.F., Poon, W.S.: Metastatic solitary fibrous tumor of the meninges. Case report. J Neurosurg 2000; 93: 490-493.

34. Pakasa, N.M., Pasquier, B., Chambonniere, M.L., et al.: Atypical presentations of solitary fibrous tumors of the central nervous system. An analysis of unusual clinicopathological and outcome patterns in three new cases with a review of the literature. Virchows Arch. 2005 Jul; 447: 81-86. Epub 2005 May 31.

35. Pérez-Núñez, A., Rivas, J.J., Ricoy, J.R., et al.: Solitary fibrous tumor of the tentorium cerebelli. Case report. J Neurosurg Sci. 2004; Jun 48: 59-62.

36. Perry, A., Scheithauer, B.W., Nascimento, A.G.: The immunophenotypic spectrum of meningeal hemangiopericitoma. A comparison with fibrous meningioma and solitary fibrous tumor of meninges. Am J Surg Pathol 1997; 21: 13541360.

37. Prayson, R.A., McMahon, J.T., Barnett, G.H.: Solitary fibrous tumor of the meninges. Case report and review of the literature. J Neurosurg 1997; 86: 1049-1052.

38. Slavik, T., Bentley, R.C., Gray, L., Fuchs, H.E., McLendon, R.E.: Solitary fibrous tumor of the meninges occurring after irradiation of a mixed germ cell tumor of the pineal gland. Clin Neuropathol 1998; 17: 55-60.

39. Suster, S., Nascimento, A.G., Miettinen, M., Nickel, J.Z., Moran, C.A.: Solitary fibrous tumors of soft tissue. Am J Surg Pathol 1995; 19: 1257-1266.

40. Suzuki, S.O., Fukui, M., Nishio, S., Iwaki, T.: Clinicopathological features of solitary fibrous tumor of the meninges. An immunohistochemical reappraisal of cases previously diagnosed to be fibrous meningioma or hemangiopericytoma. Pathol Int 2000; 50: 808-817.

41. Tihan, T., Viglione, M., Rosenblum, M.K., Olivi, A., Burger, P.C.: Solitary fibrous tumors of the central nervous system. Clinicopathological review of 18 cases and comparison with meningeal hemangiopericytoma. Arch Pathol Lab Med 2003; 27: 432- 439. 
42. Vallat-Decouvelaere, A.V., Dry, S.M., Fletcher, C.D.M.: Atypical and malignant solitary fibrous tumors in extrathoratic locations. Evidence of their comparability to intra-thoracic tumors. Am J Surg Pathol 1998; 22: 1501-1511.

43. Vorster, S.J., Prayson, R.A., Lee, J.H.: Solitary fibrous tumor of the thoracic spine. Case report and review of the literature. J Neurosurg 2000; 92 [Suppl 2]: 217-220.

44. Weiss, S.W., Nickoloff, B.J.: CD34 is expressed by a distinctive cell population in peripheral nerve, nerve sheath tumours, and related lesions. Am J Surg Pathol 1993; 17: 1039-1045.

45. Witkin, G.B., Rosai, J.: Solitary fibrous tumor of the upper respiratory tract: report of six cases. Am J Surg Patho 1991; 15: 842-848.
46. Zamecnik, M., Michal, M.: Solitary fibrous tumor (fibrous mesothelioma). Report of extraserous location. (in Slovak) Cesk Patol 1998; 34: 58-62.

Saceda-Gutiérrez, J.M.; Isla-Guerrero; A.J.; Pérez-López, C.; Ortega-Martínez, R.; Gómez de la Riva, A.; GandiaGonzález, M.L.; Gutiérrez-Molina, M.; Rey-Herranz, J.A.: Tumor fibroso solitario meníngeo: tres casos con seguimiento de 7 años y revisión de la literatura. Neurocirugía 2007; 18: 496-504.

Correspondencia postal: J. M. Saceda Gutiérrez. Servicio de Neurocirugía. $2^{a}$ planta Hospital General La Paz. Paseo de la Castellana 261, 28046 Madrid. 